Article

\title{
Sarcodia suieae acetyl-xylogalactan down-regulate RAW264.7 macrophage apoptosis factors Ddit3 and Hyou1 expression un- der heat stress
}

\author{
Kuang-Teng Wang ${ }^{1}$ Po-Kai Pan ${ }^{1}$, Tsung-Meng $\mathrm{Wu}^{1}{ }^{1}$, and $\mathrm{Yu}-\mathrm{Sheng} \mathrm{Wu}^{1}{ }^{1}$, \\ 1. Department of Aquaculture, National Pingtung University of Science and \\ Technology, Pingtung, Taiwan \\ *1. Ph.D. Tsung-Meng Wu (Department of Aquaculture, National Pingtung University of Science and Technol- \\ ogy, 1, Shuefu Road, Neipu, Pingtung 91201, Taiwan.) \\ * 2. Ph.D. Yu-Sheng Wu (Department of Aquaculture, National Pingtung University of Science and Technol- \\ ogy, 1, Shuefu Road, Neipu, Pingtung 91201, Taiwan.) \\ Phone: +886-8-7703202\#6223 \\ E-mail address: 1. wzm@mail.npust.edu.tw ; 2.wuys0313@mail.npust.edu.tw
}

\begin{abstract}
We aimed to evaluate the protective effects of acetyl-xylogalactan on the activity of RAW264.7 macrophages against heat stress. To this end, we assessed cell survival, phagocytic activity, intracellular $\mathrm{Ca}^{2+}$ level, mitochondria potential exchange, apoptotic assay findings, galactosidase activity and the RNA-seq by NGS and real-time polymerase chain reaction (PCR) expression.

In our evaluation of macrophage morphology at $37^{\circ} \mathrm{C}$ and $41^{\circ} \mathrm{C}$, the macrophages showed an oval shape at $41^{\circ} \mathrm{C}$, unlike the spindle shape at $37^{\circ} \mathrm{C}$. Therefore, $41^{\circ} \mathrm{C}$ was chosen as the heat stress condition. Subsequently, we designed an experiment to evaluate changes in the RAW264.7 macrophages after acetyl-xylogalactan treatment under heat stress. The survival of RAW264.7 macrophages treated with acetyl-xylogalactan was higher than that of controls that were not treated with acetyl-xylogalactan. Moreover, on the basis of the results of the annexin- $\mathrm{V}$ detection assay, the apoptotic activity of macrophages appeared to have reduced after treatment with acetyl-xylogalactan. Moreover, treatment with acetyl-xylogalactan resulted in a stronger recovery trend in the intracellular $\mathrm{Ca}^{2+}$ and mitochondrial membrane potential after heat stress.
\end{abstract}

RNA sequencing and real-time polymerase chain reaction (PCR) illustrated that Sarcodia suieae acetyl-xylogalactan could upregulate the expression of the anti-apoptosis Cflar gene and downregulate the expression of the apoptosis factors Ddit3, and Hyou1 to protect macrophages under heat stress.

Keywords: Sarcodia suieae, Acetyl-xylogalactan, RAW264.7, Heat stress, Apoptosis

\section{Introduction}

Hyperthermia is a major clinical manifestation of chronic and acute illnesses [1]. During hyper-inflammatory responses, interactions of the immune cellular and micro-environments can help minimize impending injury or infection and restore tissue homeostasis. However, an uncontrolled inflammation response may lead to a chronic inflammatory disease [2]. For example, inflammatory responses play a role in the pathogenesis of autoimmune diseases and increase the permeability of the vascular endothelium, allowing leakage of serum components and extravasation of immune cells [3]. In the inflammatory process, which is characterized by redness, swelling, heat, pain, and loss of tissue function [3], the hyperthermia enhances the activity of reactive oxygen species (ROS), resulting in damage to cellular proteins and DNA damage [4]. This phenomenon is known as heat 
stress. Heat stress in the cellular response is known to reduce alterations in host physiological and immune systems, which are associated with cellular death, impaired growth [5], and enhanced mucosal damage [6].

Heat stress can lead to multiple forms of DNA damage, such as base damage, singlestrand breaks, replication inhibition, and double-strand breaks [7]. Heat stress is known to be accompanied by apoptotic responses and autophagic signaling [8]. Heat stress induced by $12 \mathrm{~h}$ of hyperthermia can cause apoptotic signaling [9].

To our knowledge, heat stress can induce cell apoptosis via phosphorylation of the eIF2 $\alpha$ to protect other cells [10]. Moreover, cytokine-treated human islets have been reported to regulation of pro-apoptotic genes through NF-kappa B [11] (Sarkar et al., 2009). Our previous study already showed that acetyl-xylogalactan extracted from Sarcodia suieae could activate RAW264.7 macrophages via NF-kappa B [12]

Therefore, this study aimed to evaluate the protective effects of Sarcodia suieae acetylxylogalactan on macrophages under heat stress.

\section{Results}

\subsection{RAW264.7 macrophage survival under heat stress}

RAW264.7 macrophages were incubated at $37^{\circ} \mathrm{C}$ and $41^{\circ} \mathrm{C}$, and the cells showed an oval shape at $41^{\circ} \mathrm{C}$, which differed from the spindle shape shown at $37^{\circ} \mathrm{C}$, as presented in Figure. 1C. On the basis of these findings, the heat stress temperature was chosen as $41^{\circ} \mathrm{C}$. In the cell survival experiment, RAW264.7 macrophages were treated with 10, 20, and $30 \mu \mathrm{g} / \mathrm{mL}$ acetyl-xylogalactan for 12 and $24 \mathrm{~h}$ at $41^{\circ} \mathrm{C}$, and the survival of the treatment groups at $41^{\circ} \mathrm{C}$ was higher than those in the control.

In this analysis, the 20 and $30 \mu \mathrm{g} / \mathrm{mL}$ treatment groups showed significantly greater survival than the control $(\mathrm{p}<0.05)$ at $12 \mathrm{~h}$, as shown in Figure. $1 \mathrm{~A}$. At $24 \mathrm{~h}$, the $30 \mu \mathrm{g} / \mathrm{mL}$ treatment group showed significantly greater survival than the control $(\mathrm{p}<0.05)$. On the basis of these findings, we thought that the Sarcodia suieae acetyl-xylogalactan could maintain RAW264.7 macrophage survival under the heat stress.

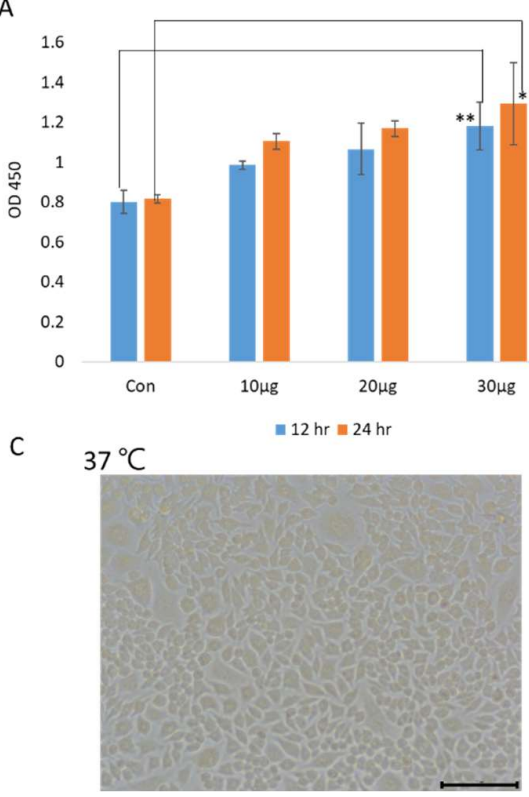

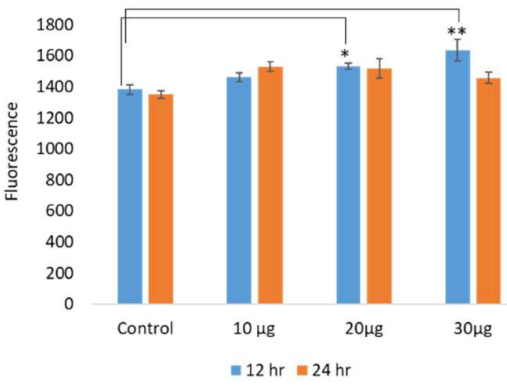

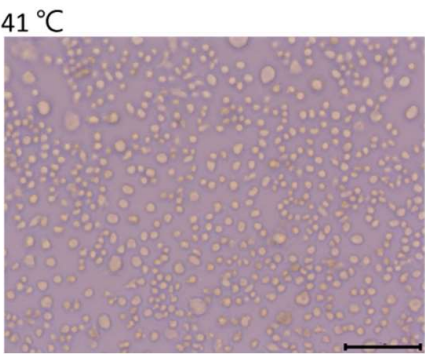

Figure 1. RAW 264.7 macrophage survival. (A) $30 \mu \mathrm{g} / \mathrm{mL}$ treatment groups showed significantly greater survival than the control $(\mathrm{p}<0.01)$ at $12 \mathrm{~h}$ and $24 \mathrm{~h}(\mathrm{p}<0.05)$ under the heat. (B) acetyl-xylogalactan treatment could significantly induce phagocytic ability $(\mathrm{p}<$ 0.05 ) at $12 \mathrm{~h}$ under heat stress but not at $24 \mathrm{~h}$. (C) the Raw 264.7 cells showed an oval shape at $41^{\circ} \mathrm{C}$, which differed from the spindle shape shown at $37^{\circ} \mathrm{C}$. 


\subsection{RAW264.7 macrophage phagocytic activity under heat stress}

The results presented in Figure. 1B show that acetyl-xylogalactan treatment could significantly induce phagocytic ability $(\mathrm{p}<0.05)$ at $12 \mathrm{~h}$ under heat stress. However, at $24 \mathrm{~h}$, the phagocytic ability did not differ between groups $(\mathrm{p}>0.05)$.

Thus, RAW264.7 macrophages treated with acetyl-xylogalactan could maintain their phagocytic ability under heat stress for $12 \mathrm{~h}$.

\subsection{RAW264.7 macrophage apoptosis under heat stress}

The results in Figure. 2 A show that $20 \mu \mathrm{g} / \mathrm{mL}$ acetyl-xylogalactan treatment could significantly reduce apoptosis $(\mathrm{p}<0.05)$ at $12 \mathrm{~h}$ under heat stress. At $24 \mathrm{~h}$ under heat stress, apoptosis did not show significant differences among groups ( $p>0.05)$, as shown in Figure. 2B.

Thus, acetyl-xylogalactan treatment could reduce macrophage apoptosis under heat stress for $12 \mathrm{~h}$.

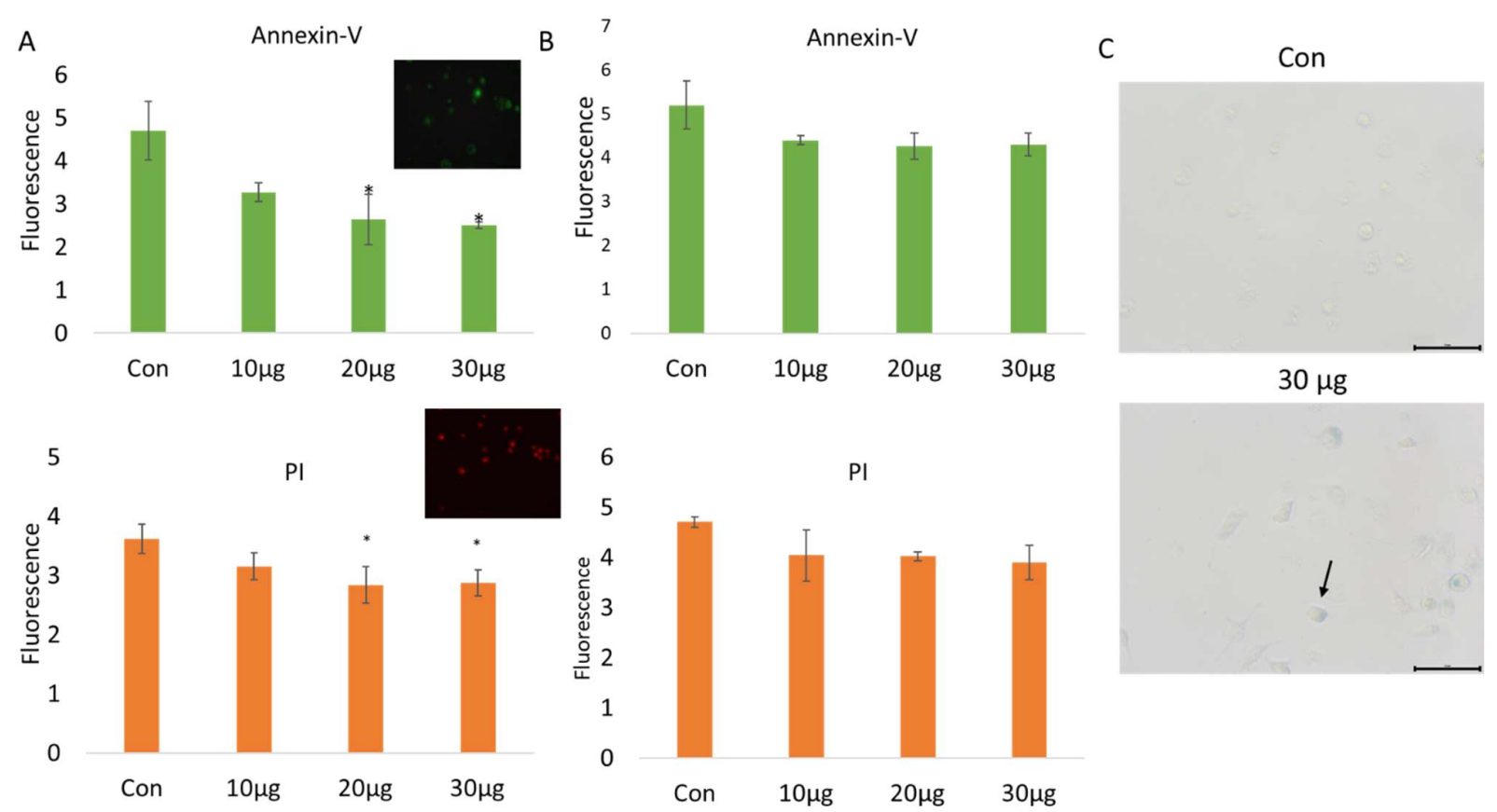

Figure 2. RAW 264.7 macrophage apoptosis detection via Annexin-V. (A) $20 \mu \mathrm{g} / \mathrm{mL}$ acetyl-xylogalactan treatment could significantly reduce apoptosis $(\mathrm{p}<0.05)$ at $12 \mathrm{~h}$ under heat stress. (B) However, apoptosis did not show significant differences among groups at 24h ( $\mathrm{p}>0.05)$. (C) RAW 264.7 macrophage galactosidase detection. $30 \mu \mathrm{g} / \mathrm{mL}$ acetyl-xylogalactan significantly induce galactosidase production (blue-stained cells) at $12 \mathrm{hr}$ under $41^{\circ} \mathrm{C}$. (Scale bar was as $50 \mu \mathrm{m}$ )

\subsection{RAW264.7 macrophage galactosidase production under heat stress}

The findings in Figure. 2C show that treatment with $30 \mu \mathrm{g} / \mathrm{mL}$ acetyl-xylogalactan could significantly induce galactosidase production (blue-stained cells) at $12 \mathrm{hr}$ under $41^{\circ} \mathrm{C}$ heat stress. In contrast, galactosidase production was not observed in the control group.

Intracellular $\mathrm{Ca}^{2+}$ concentration and mitochondrial membrane potential after heat stress. RAW264.7 intracellular Ca2+ concentration recovery is presented in Figure. 3A, 
which shows that there was no significant change $(p>0.05)$ while pre-treatment with various concentrations of the acetyl-xylogalactan for $24 \mathrm{~h}$, following incubation under heat stress. However, the macrophage mitochondrial membrane potential was recovery to lower than that in the control $(\mathrm{p}<0.01)$, as shown in Figure. 3B.

Thus, treatment with acetyl-xylogalactan increased the RAW264.7 mitochondrial membrane potential recovery to reduce intracellular stress. We thought that the acetylxylogalactan could regulate the macrophage intracellular stress induced by the elevated temperature.

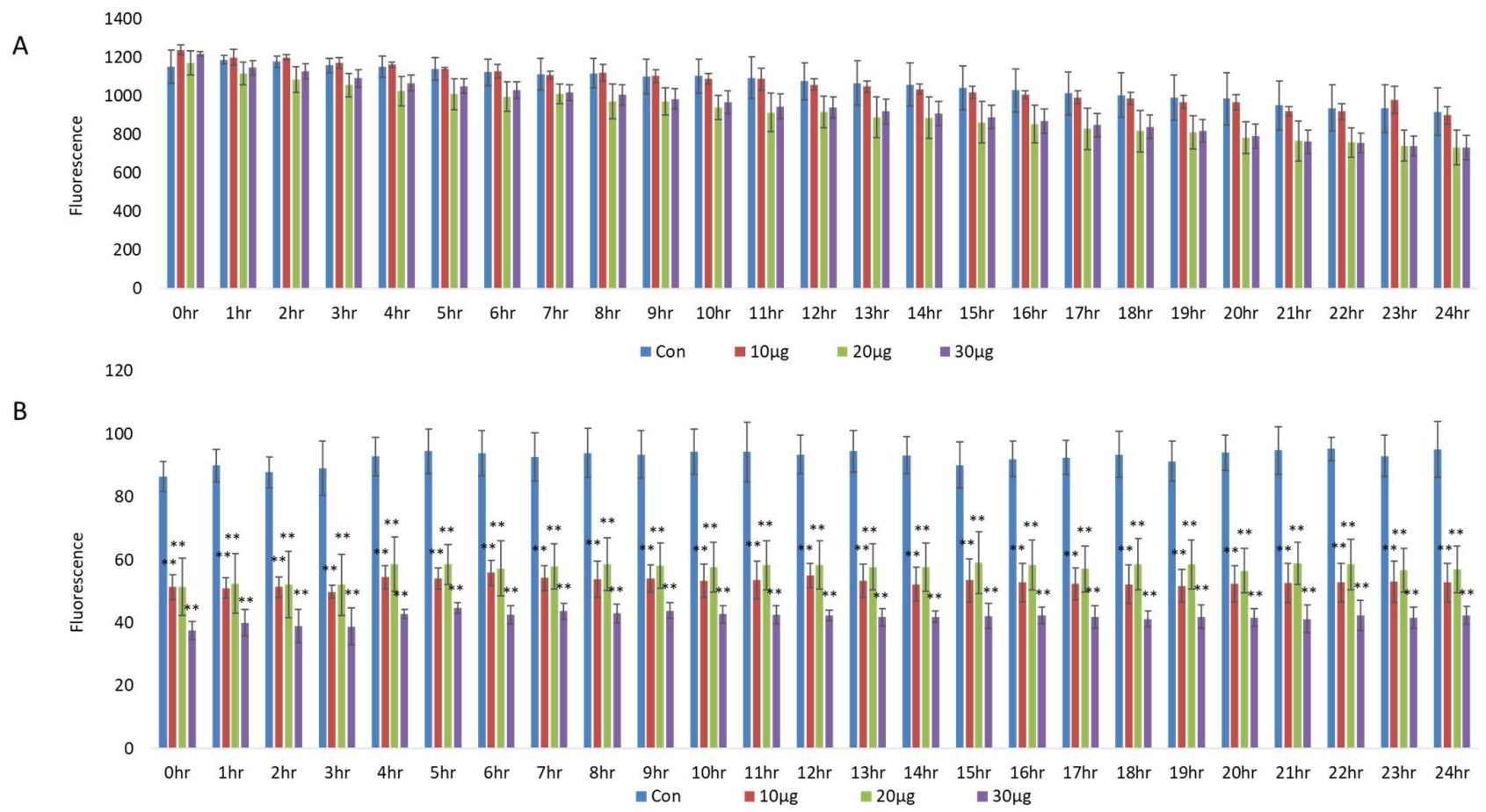

Figure 3. RAW 264.7 macrophage intracellular Ca2+ concentration and mitochondria membrane potential. (A) there was no significant change $(p>0.05)$ while pre-treatment with various concentrations of the acetyl-xylogalactan for $24 \mathrm{~h}$, following incubation under $41^{\circ} \mathrm{C}$. (B) mitochondrial membrane potential was recovery to lower than that in the control $(\mathrm{p}<0.01)$.

\subsection{RNA sequencing (Transcriptome)}

Macrophage RNA was isolated and mapped to the reference genome. RNA-seq analysis showed that RNA and gene expression significantly changed with acetyl-xylogalactan treatment. After the sequencing was completed, the predicted pathway of DEG analysis, the acetyl-xylogalactan may affect the counts of the apoptosis gene as 10 as presented in the KEGG and GO term analysis as Figure. $4 \mathrm{~A}$ and B. To this analysis, it was known to regulate the intrinsic apoptosis. As the $\log 2$ fold change analysis of the NGS, It was exactly observed that the 10, 20 and $30 \mu \mathrm{g}$ treatment was able to regulate the significant difference as Figure. $4 \mathrm{C}$ and D. Compared to the control, the volcano (Figure. $4 \mathrm{D})$ figure was shown to induce the gene expression with the concentration treated. 
A

DEG All KEGG Enrichment Pathways Barplot

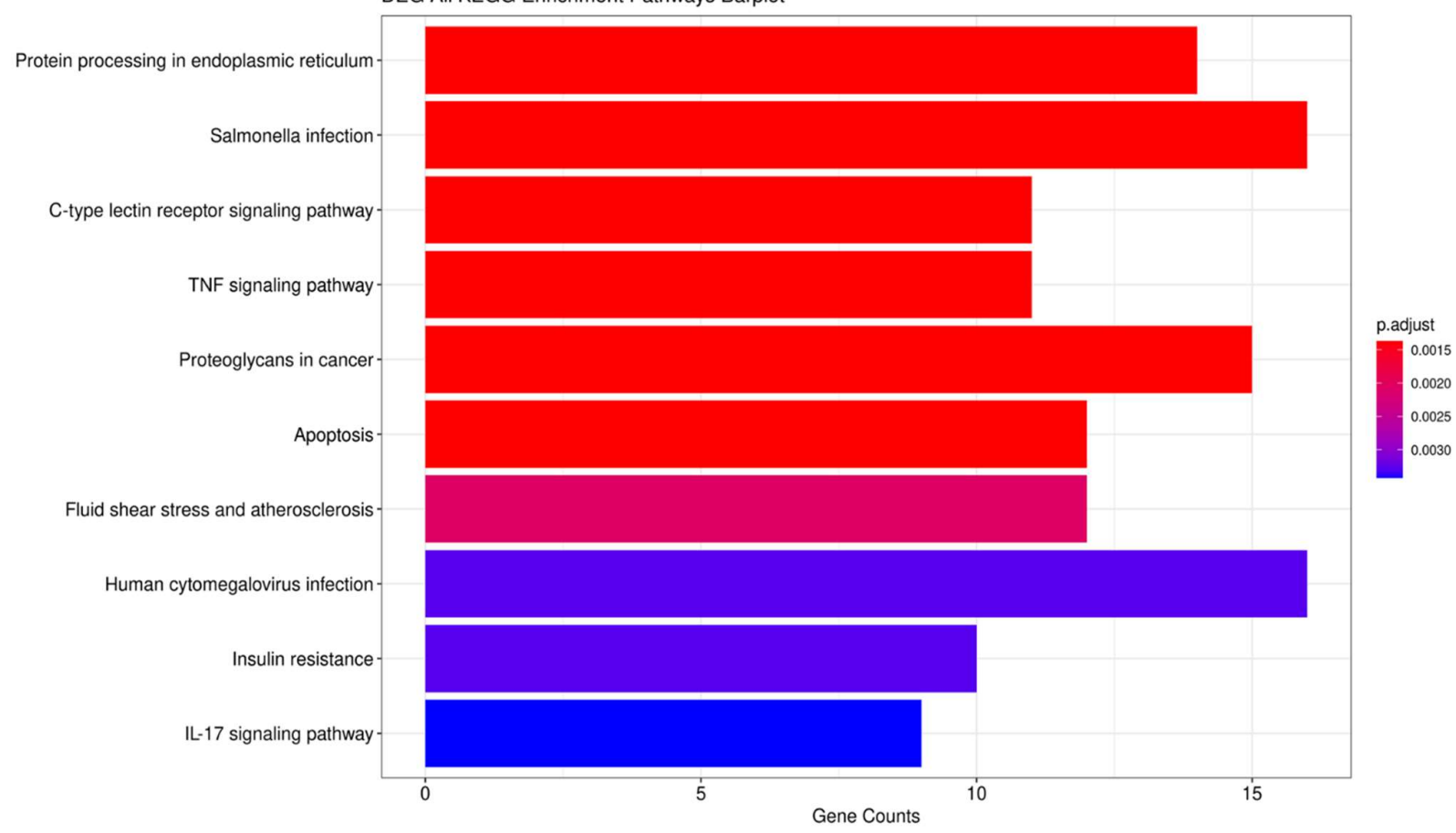

B

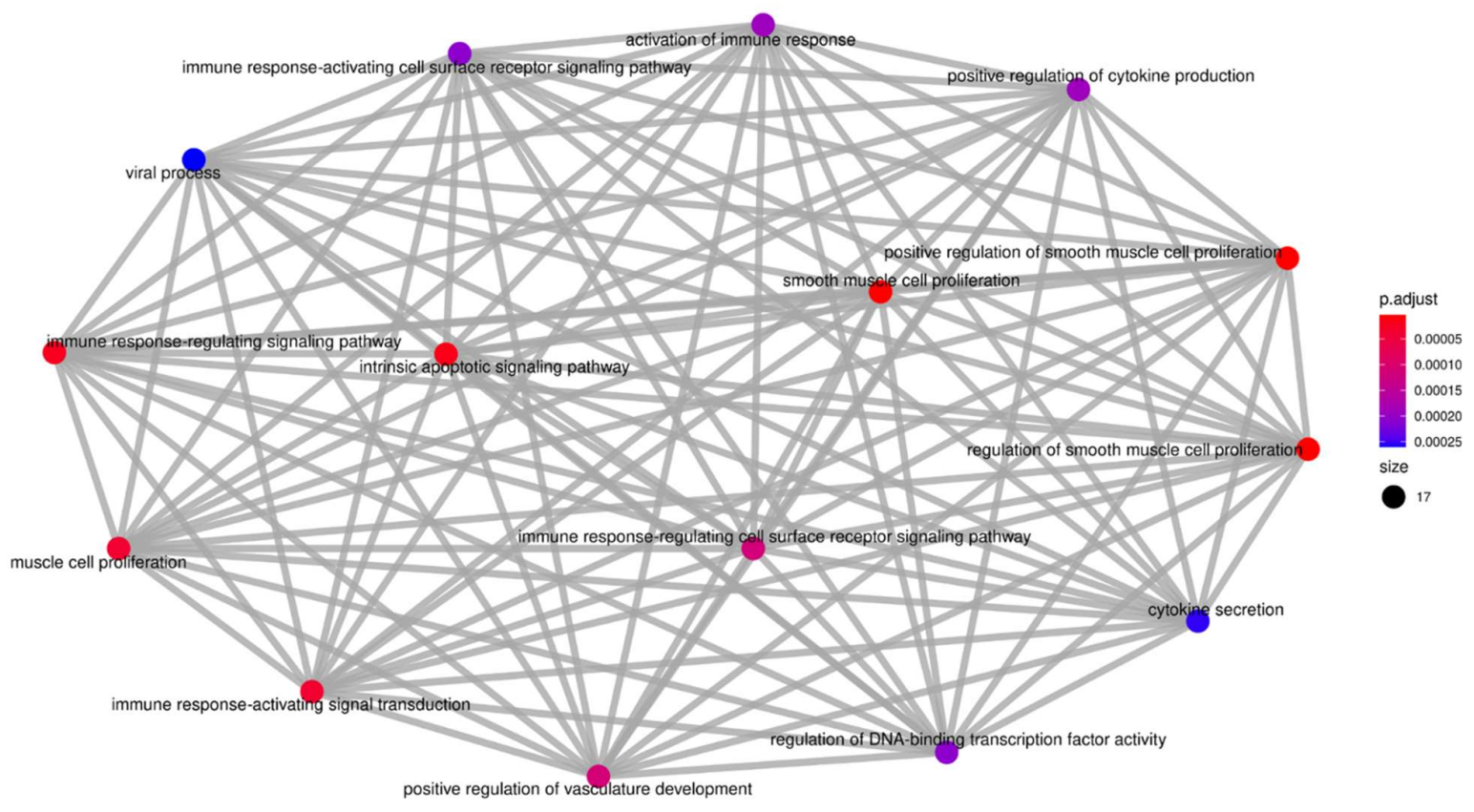

C
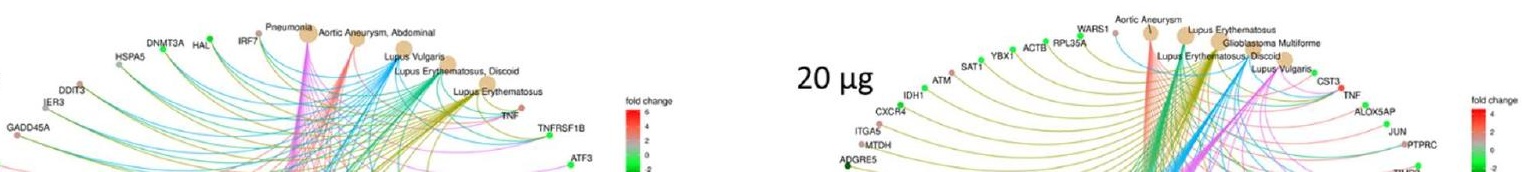


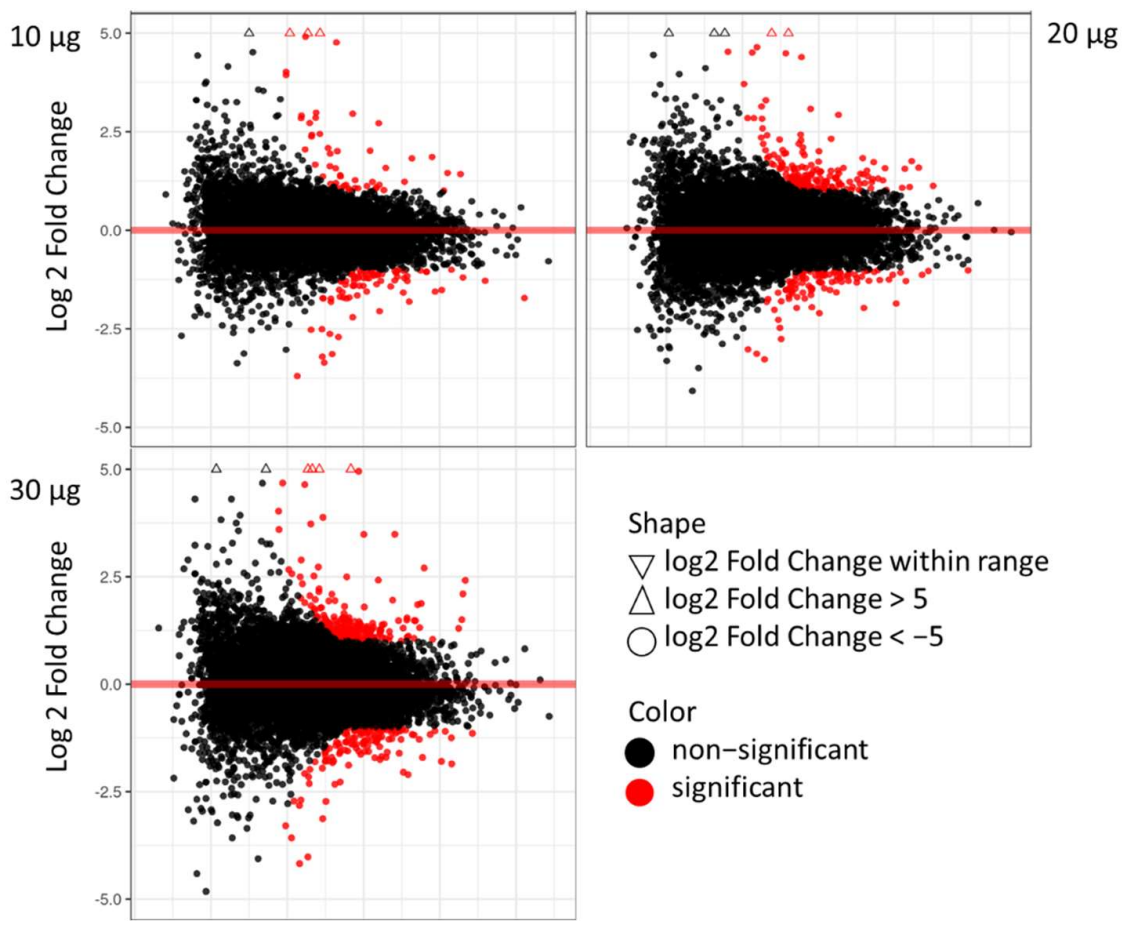

Figure 4. RNA sequencing (Transcriptome) analysis. (A) acetyl-xylogalactan may affect the counts of the apoptosis gene as 10 as presented in the KEGG analysis. (B) acetyl-xylogalactan may regulate the relative cell phenomenon. (C) Log 2 fold change of the acetyl-xylogalactan various concentration treatment (D) Volcano map of the acetyl-xylogalactan various concentration treatmen

According to the NGS analysis, the proposed gene as Nfkbia, Ddit3, Gadd45a, Akt3, Hyou 1 and Cflar were examined. The results showed that acetyl-xylogalactan down-regulate apoptosis under heat stress via target genes such as Nfkbia, Ddit3, Gadd45a, Akt3, 
and Hyou1 (Figure. 5A and B). In the Figure. 5C of the Gene Ontology (GO) analysis, it was exactly pointed that treatment with acetyl-xylogalactan was able to regulate the RAW 264.7 intrinsic apoptotic signalling pathway under $41^{\circ} \mathrm{C}$. However, in the Cflar expression, acetyl-xylogalactan was shown to up-regulate the expression. Taken together, these findings indicate that $S$. suieae acetyl-xylogalactan treatment reduced the expression of apoptosis factors under the heat stress, as shown in Figure. 5D.

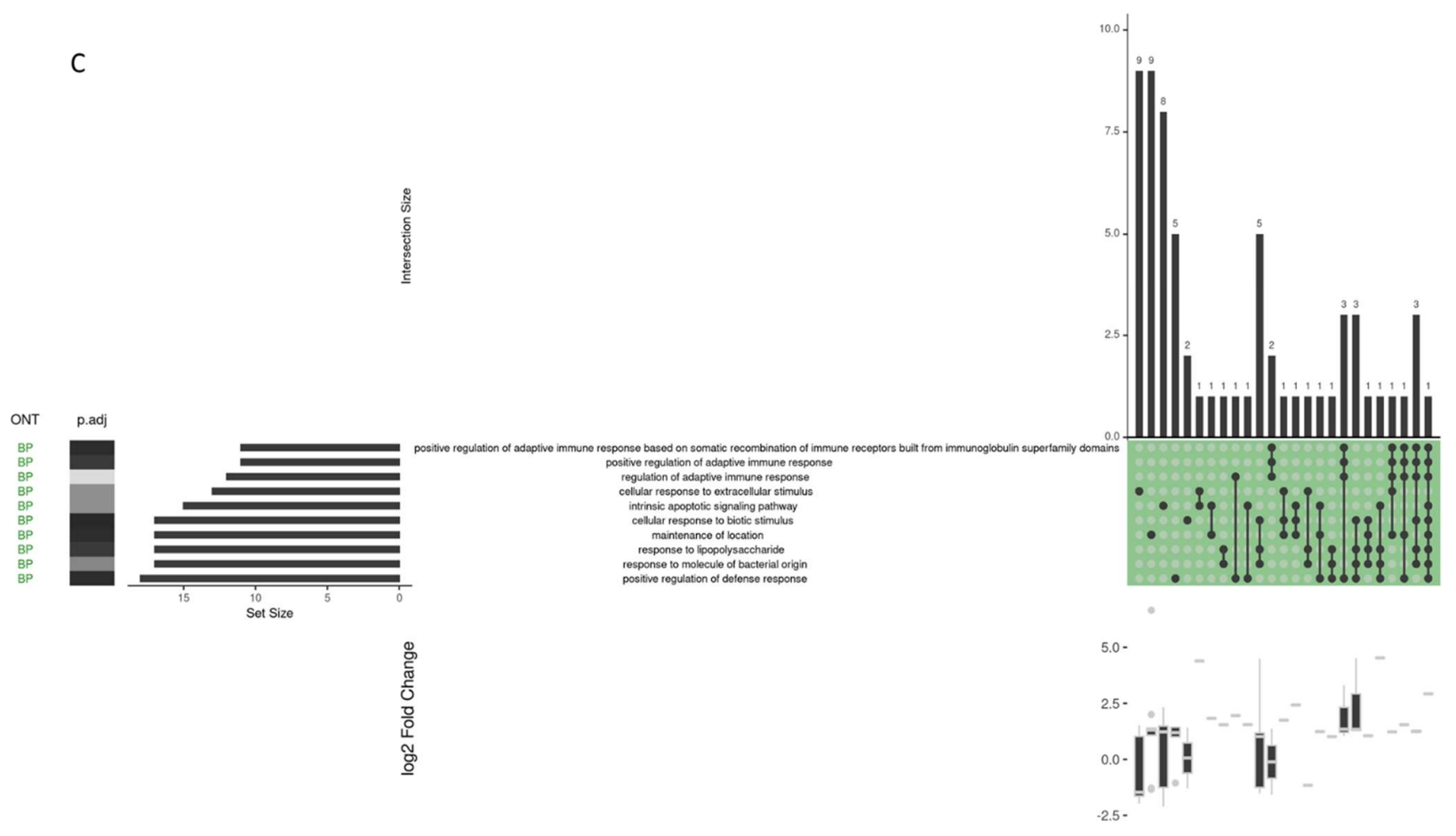


D
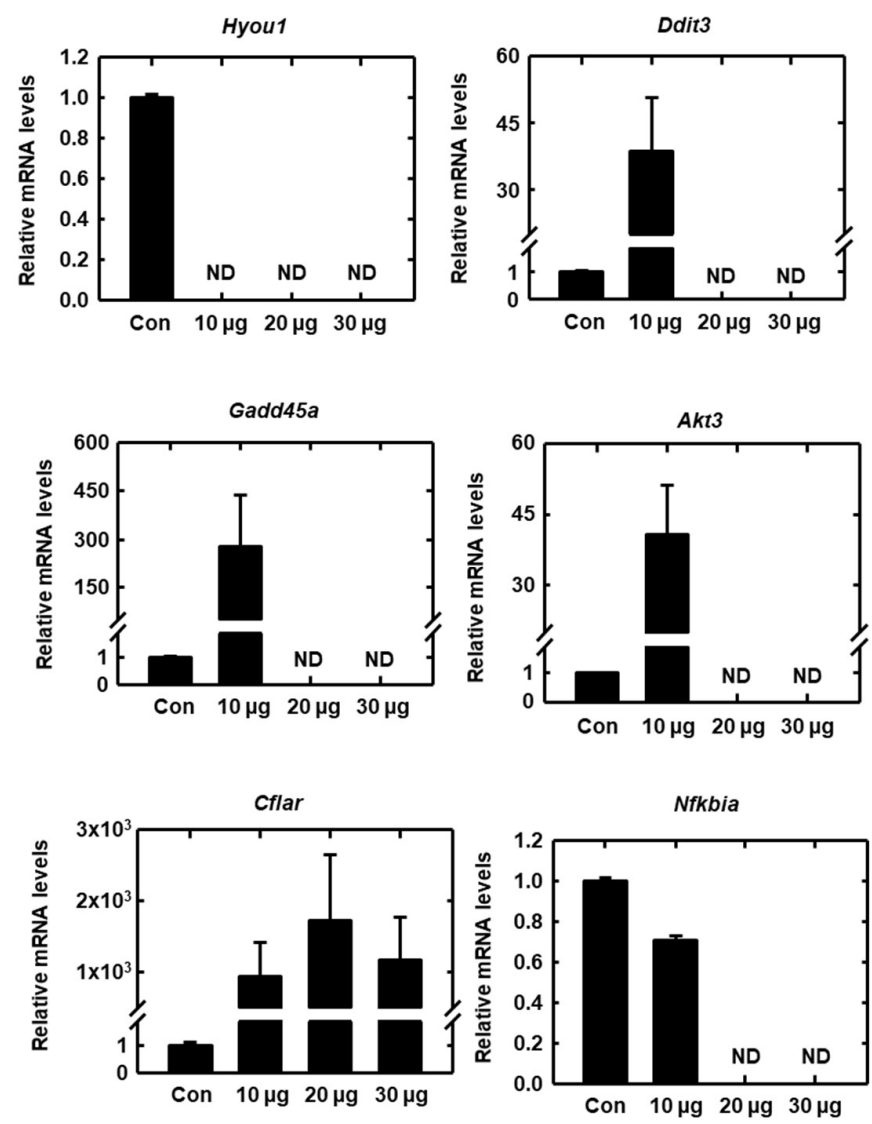

Figure 5. (A) The network of the relative RNA-seq analysis. (B) The heatmap of the RNA-seq analysis, which was presented with log 2-Fold change. (C) Gene Ontology (GO) analysis. (D) mRNA expression. acetyl-xylogalactan down-regulate apoptosis under heat stress via target genes such as Nfkbia, Ddit3, Gadd45a, Akt3, and Hyou1 and up-regulate the Cflar expression.

\section{Discussion}

Heat stress (HS) is known to induce a significant increase in cytosolic $\mathrm{Ca}^{2+}$ concentration. Further evidence has shown that $\mathrm{Ca}^{2+}$ might be a factor in the regulation of ganoderic acid (GA) biosynthesis and the accumulation of heat shock proteins (HSP) production [13]. A previous study evaluated the response of a chicken macrophage-like (HD11) cell line to heat stress and LPS stimulation and showed that the monocyte/macrophage cell line responded to heat stress with increased mRNA abundance of the HSP [14]. One study revealed that heat stress (HS) induced apoptosis of vascular endothelial cells associated with reactive oxygen species (ROS)-induced p53 translocation into mitochondria to induce changes in intracellular $\mathrm{Ca}^{2+}$ levels [15]. Intracellular $\mathrm{Ca}^{2+}$ influx through cation channels, mitochondrial function, and oxidative stress induced parallel pathophysiological mechanisms in diabetic neuropathy. Impaired insulin signaling and $\mathrm{Ca}^{2+}$ influx through TRP and voltage-gated $\mathrm{Ca}^{2+}$ channel activations triggers sensory neuron mitochondrial depolarization [15].

In our study, we found that acetyl-xylogalactan could recover macrophage intracellular $\mathrm{Ca}^{2+}$ after heat stress and downregulated HSP gene expression. Moreover, assessment of mitochondrial membrane potential indicated recovery of the membrane potential after treatment with acetyl-xylogalactan under heat stress. This phenomenon was similar 
to the intracellular $\mathrm{Ca}^{2+}$ expression and apoptotic genes downregulation. We propose that treatment with acetyl-xylogalactan could inhibit intracellular stress to reduce mitochondrial-mediated damage signaling leading to the cell death.

In the previous study, we examined whether the acetyl-xylogalactan affected on the macrophage phagocytic ability without heat stress at $37{ }^{\circ} \mathrm{C}$. To the published evidence, high concentration of $30 \mu \mathrm{g} / \mathrm{ml}$ was able to increase the phagocytic ability during $12 \mathrm{hr}$ treatment, however it was not with an enhancing trend after $24 \mathrm{hr}$ treatment as the reference [12]. Based on that, this research, we examined to observe whether the phagocytic ability exchange under the heat stress with or without the acetyl-xylogalactan. As this result shown, the 20 and $30 \mu \mathrm{g} / \mathrm{ml}$ treatment was also to maintain the phagocytic ability similar under the heat stress. However, the control group without treatment, the ability was with a reducing trend under the heat stress.

By this evidence, we observed that treating with high concentration of 20 and 30 $\mu \mathrm{g} / \mathrm{ml}$, the Annexin-V staining reading data was lower than the control and $10 \mu \mathrm{g} / \mathrm{ml}$, this evidence was also similar to the PI-staining data at $12 \mathrm{hr}$. However, at $24 \mathrm{hr}$ observation, the Annexin-V staining or PI- staining data was presented with no significant difference, we thought that treating with the acetyl-xylogalactan 20 and $30 \mu \mathrm{g} / \mathrm{ml}$, the protective ability was just for $12 \mathrm{hr}$. With a long term exposure of the heat stress $\left(41^{\circ} \mathrm{C}\right)$, the apoptotic was also presented in the high concentration treatment group.

Polymorphonuclear cells and peripheral blood mononuclear cells (PBMCs) under heat stress are known to show cellular apoptosis at $41^{\circ} \mathrm{C}$ [16]. The intracellular $\mathrm{Ca}^{2+}$ level is also related to the cell apoptosis rate via down-regulation of FKBP12.6 expression and up-regulation of phosphorylated-Ser2814-RyR2 and cleaved caspase3 expression [17]. A recent study pointed out that mitochondria play a central role in apoptosis, and $\mathrm{Ca}^{2+}$ overload is believed to prime the mitochondrial permeability transition pore (mPTP) to open at the stage of myocardial reperfusion, with the intracellular $\mathrm{Ca} 2+$ levels detected by laser scanning confocal microscopy at $48 \mathrm{~h}$ following transfection [18]. In our study, RAW264.7 treated with acetyl-xylogalactan at $41^{\circ} \mathrm{C}$ showed reduced cellular apoptosis. However, the Sarcodia suieae acetyl-xylogalactan was able to reduce RAW264.7 macrophage apoptosis only for $12 \mathrm{~h}$ under the heat stress.

TRAIL and TRD have been shown to induce apoptotic cell death and significantly decreased proliferation in HT1080 cells. The expression of several genes have been shown to be related to apoptotic pathways, such as Nfkbia. TRAIL effectively induced apoptotic cell death in HT1080 fibrosarcoma cells by upregulating the Arhgdia, Tnfaip3, and Nfkbia more than two-fold in comparison with the control [19]. CNPs were also shown to enhance early apoptosis in hMSCs. Similarly, earlier studies demonstrated that titanium dioxide, cerium dioxide, and zinc oxide nanoparticles stimulated apoptosis in different cells

The DNA damage-inducible transcript 3, also known as C/EBP homologous protein $(\mathrm{CHOP})$, is known to be a pro-apoptotic transcription factor. Ddit3 and putative AP-1 have been putatively identified in the TNFRSF10A promoter, which regulates the extrinsic pathway of apoptosis [20,21]. Animals deficient in both Jun and Ddit3 were generated and evaluated after mechanical optic nerve injury. Combined deficiency of Jun and Ddit3 was more protective of RGCs after axonal injury than either Jun or Ddit3 deficiency alone, and provided profound long-term protection of RGCs after axonal injury [22]. Research has already illustrated that the long-isoform Cflar plays a critical role in all three fundamental intracellular processes - autophagy, necroptosis, and apoptosis in T lymphocytes [23] and controls not only the classical death receptor-mediated extrinsic apoptosis pathway, but also the non-conventional pattern recognition receptor-dependent apoptotic pathway [24]. To our knowledge, Cflar, an inhibitor of apoptosis, regulates apoptosis in vivo, and Cflar up-regulation could induce the resistance of HepG2 cells against Taxolinduced apoptosis [25]. To our evidence, Sarcodia suieae acetyl-xylogalactan enhanced Cflar gene expression, suggesting that Sarcodia suieae acetyl-xylogalactan induced antiapoptosis factors to protect RAW264.7 cells against heat-induced apoptosis. Further, we 
analyzed the RNA sequencing data of RAW264.7 cells by the NGS and proposed a pathway of reduced apoptosis-related genes. As confirm, we detected apoptosis-related genes such as Nfkbia, Ddit3, Gadd45a, Akt3, and Hyou1.

This study investigated the cellular functions of RAW 264.7 macrophages treated with S. suieae acetyl-xylogalactan under heat stress. In the RNA-seq analysis, the S. suieae acetyl-xylogalactan was demonstrated to have positively regulated apoptosis, phagosome production and IL-17 signaling etc., based on the observed KEGG pathway (As shown in the Supplement Figure. 2). Of the predicted target genes in the DEG seq prediction demonstrating $S$. suieae acetyl-xylogalactan facilitated the regulation of the apoptotic relative factors. Predicted expressed genes included Nfkbia, Hyou1, Ddit3, Gadd45a, Akt3, and Cflar (As shown in the Supplement Table 3).

\section{Materials and Methods}

\subsection{Sarcodia suieae acetyl-xylogalactan preparation}

The preparation method for Sarcodia suieae acetyl-xylogalactan has been reported in a previous study [12], and this technique was used in the present study. Briefly, dried Sarcodia suieae was treated in water at $60^{\circ} \mathrm{C}$ for 6 hours. The extracted acetyl-xylogalactan contained galactose $(91 \%)$ and xylose $(9 \%)$ as monosaccharides, up to $80.6 \%$ polysaccharides, and 19.3\% acetyl content in NMR and HPLC analysis. The molecular weight of the acetyl-xylogalactan was $88.5 \mathrm{kDa}$.

\subsection{Cellular findings}

4.2.1 Survival of RAW264.7 cells under heat stress

To examine the toxicity of Sarcodia suieae acetyl-xylogalactan on the RAW264.7 macrophages, $1 \times 10^{6}$ RAW264.7 macrophages were treated without or with Sarcodia suieae acetyl-xylogalactan at concentrations of 10,20 , and $30 \mu \mathrm{g} / \mathrm{mL}$ for 12 and $24 \mathrm{~h}$ at $41^{\circ} \mathrm{C}$ under $5 \% \mathrm{CO}_{2}$. At the end of this treatment, a Cell counting Kit-8 (CCk-8) (B34302, bimake) was used to examine cell survival with a microplate reader at OD $450 \mathrm{~nm}$.

\subsubsection{Apoptosis of RAW264.7 macrophages under heat stress}

To examine whether Sarcodia suieae acetyl-xylogalactan protected RAW264.7 macrophages against apoptosis, $1 \times 10^{6}$ RAW264.7 cells were cultured without (control) and with Sarcodia suieae acetyl-xylogalactan at concentrations of 10, 20, $30 \mu \mathrm{g} / \mathrm{mL}$ for 12 and 24 $\mathrm{h}$ at $41^{\circ} \mathrm{C}$ under $5 \% \mathrm{CO}_{2}$. At the end of the treatment period, an Annexin V-FITC Apoptosis Detection kit (AVK250, Strong Biotech Corporation) was used to measure the degree of apoptosis by the fluorescence microplate reader.

\subsubsection{Phagocytic activity of RAW264.7 cells under heat stress}

The phagocytic activity of RAW264.7 cells was determined by the EZ CellTM Phagocytosis Assay kit (Green E.coli). Briefly, $1 \times 10^{6}$ RAW264.7 cells were cultured without (control) and with 10, 20, and $30 \mu \mathrm{g} / \mathrm{mL}$ of Sarcodia suieae acetyl-xylogalactan and incubated at $41^{\circ} \mathrm{C}$ under $5 \% \mathrm{CO}_{2}$ for 12 and $24 \mathrm{~h}$. Subsequently, the culture medium was removed and phagocytic activity was detected at Ex/Em $=490 / 520$.

\subsubsection{Observation of galactosidase expression in RAW264.7 cells under heat stress}

The expression of galactosidase in RAW264.7 cells was monitored by the Senescent Detection Kit (Cat. K320-250, Biovision) under a microscope. Briefly, $1 \times 10^{6}$ RAW264.7 cells were cultured with or without 10, 20,30 $\mu \mathrm{g} / \mathrm{mL}$ of Sarcodia suieae acetyl-xylogalactan and incubated at $41^{\circ} \mathrm{C}$ under $5 \% \mathrm{CO}_{2}$ for $12 \mathrm{~h}$. Subsequently, the culture medium was removed and observed under microscope.

\subsubsection{Mitochondrial membrane potential changes in RAW264.7 cells after heat stress}

To analyze mitochondrial membrane potential changes in RAW264.7 cells, $1 \times 10^{6}$ RAW264.7 cells were cultured without (control) or with 10, 20, $30 \mu \mathrm{g} / \mathrm{mL}$ Sarcodia suieae 
acetyl-xylogalactan and incubated for 12 or $24 \mathrm{~h}$ at $41^{\circ} \mathrm{C}$ under $5 \% \mathrm{CO} 2$. At the end of the incubation period, the culture medium was removed and the mitochondrial membrane potential changes were determined for $24 \mathrm{~h}$ by using the JC-9 Dye (Mitochondrial Membrane Potential Probe) (D22421, Thermo) as to determine the green fluorescent changes in the potential after heat stress. The green fluorescent was detected the excitation as $535 \mathrm{~nm}$, emission as $590 \mathrm{~nm}$ by using of the Spectra max Gemini XPS (Molecular Device).

\subsubsection{Intracellular $\mathrm{Ca}^{2+}$ concentration in RAW264.7 cells after heat stress}

For analysis of intracellular $\mathrm{Ca}^{2+}$ expression, control and experimental groups were formed as in the previous assessments and incubated for 12 or $24 \mathrm{~h}$ at $41^{\circ} \mathrm{C}$ under $5 \% \mathrm{CO}_{2}$. At the end of the incubation period, the culture medium was removed and intracellular $\mathrm{Ca}^{2+}$ levels were continue determined for $24 \mathrm{~h}$ by using the Fluo- 4 Direct Calcium Assay Kit (F10471; Thermo).

\subsection{Gene Expression}

4.3.1 RNA sequencing (Transcriptome)

Control and experimental groups were formed as in the previous assessments and incubated at $41^{\circ} \mathrm{C}$ under $5 \% \mathrm{CO}_{2}$ for $24 \mathrm{~h}$. RAW 264.7 macrophages $\left(1 \times 10^{6}\right.$ cells $)$ were treated with or without 10,20 , or $30 \mu \mathrm{g} / \mathrm{mL}$ S. suieae acetyl-xylogalactan for $24 \mathrm{~h}$ at $41^{\circ} \mathrm{C}$ under $5 \% \mathrm{CO}_{2}$. Thereafter, RNA was isolated from the cells using Azol RNA Isolation Reagent (Arrowtech). RNA concentrations were determined using Nanodrop, and then, $1 \mu \mathrm{g}$ of RNA was sent to Biotools Co., Ltd., Taiwan for the RNA sequencing analysis, where NovaSeq. 6000 Sequencing System (Illumina) is used.

The reference genome mapped using HISAT2. Only filtered reads could be used to analyse the mapping status of RNA-seq data to the reference genome. The original data obtained by high-throughput sequencing (Illumina NovaSeq 6000 platform) were transformed into raw sequenced reads by CASAVA base calling and stored in FASTQ format. FastQC and MultiQC were used to check fastq files for quality. The obtained raw pairedend reads were filtered by Trimmomatic (v0.38) to discard low-quality reads, trim adaptor sequences, and eliminate poor-quality bases with the following parameters: LEADING:3 TRAILING:3 SLIDINGWINDOW:4:15 MINLEN:30. The obtained high-quality data (clean reads) was used for subsequent analysis. Read pairs from each sample were aligned to the reference genome (e.g., H. sapiens, GRCh38) by the HISAT2 software (v2.1.0). Feature Counts (v1.6.0) was used to count the reads numbers mapped to individual genes. For gene expression, the "Trimmed Mean of Mvalues" normalization (TMM) was performed DEGseq (v1.36.1) without biological duplicate and the "Relative Log Expression" normalization (RLE) was performed using DESeq2 (v1.22.1) with biological duplicate. Differentially expressed genes (DEGs) analysis of two conditions was performed in $\mathrm{R}$ using DEGseq (without biological replicate) and DESeq2 (with biological replicate), which based on negative binomial distribution and Poisson distribution model, respectively. The resulting p-values were adjusted using the Benjamini and Hochberg's approach for controlling the FDR. GO and KEGG pathway enrichment analysis of DEGs were conducted using cluster Profiler (v3.10.1). The DOSE package was used to mapping of Disease Ontology (DO) terms to MeSH, ICD, NCI's thesaurus, SNOMED and OMIM. Gene set enrichment analysis (GSEA) was performed with 1,000 permutations to identify enriched biological functions and activated pathways from the molecular signatures database (MSigDB). MSigDB is a collection of annotated gene sets for use with GSEA software, including hallmark gene sets, positional gene sets, curated gene sets, motif gene sets, computational gene sets, GO gene sets, oncogenic gene sets, and immunologic gene sets. The proteinprotein interaction (PPI) network was constructed for differential expression genes using STRINGdb (https://string-db.org/). In addition, Weighted Gene Co-expression Network Analysis (WGCNA) was constructed the co-expression network based on the correlation coefficient of expression pattern using the WGCNA (v1.64) package. 
4.3.2 Real-time qPCR analysis

Real-time qPCR analysis of the macrophage Nfkbia, Ddit3, Gadd45a, Akt3, Hyou 1 and Cflar was performed by Biotools Co., Ltd. The quantitative real-time PCR service is performed by using TOOLS 2xSYBR qPCR Mix Kit (FPT-BB05). TOOLS 2xSYBR qPCR Mix Kit is specially designed to perform Real-time PCR in SYBR Green I fluorescent-based detection assays. TOOLS 2xSYBR qPCR Mix adopts a unique dual hot-start enzymes system (chemically modified HotStar Taq DNA polymerase and antibody modified Anti Taq DNA Polyerase), which, plus the pre-optimized buffer solution, provides a convenient format for highly sensitive and specific qPCR amplification.

The qPCR protocol was as below:

1: $95.0^{\circ} \mathrm{C}$ for $15: 00$

2: $95.0^{\circ} \mathrm{C}$ for $0: 10$

3: $62.0^{\circ} \mathrm{C}$ for 0:30 Plate Read

4: GOTO 2, 59 more times

5: $95.0^{\circ} \mathrm{C}$ for $0: 10$

6: Melt Curve $65.0^{\circ} \mathrm{C}$ to $95.0^{\circ} \mathrm{C}$ : Increment $0.5^{\circ} \mathrm{C} 0: 05$

7: Plate Read

Beta-actin was used as the reference in the comparative CT to determine the relative alteration. Fluorescence was analysed using the auto CT method to determine the threshold of each gene, and the $2-\Delta \Delta \mathrm{CT}$ method was used to calculate $\mathrm{CT}$ values by using StepOne (version 2.3). Data are presented as fold changes in the mRNA level normalised to the reference gene $\beta$-actin. The following oligonucleotide sequences were used for creating qPCR primers.

$\begin{array}{lll}\text { Gene } & \text { Forward primer } & \text { Reverse primer } \\ \beta \text {-actin } & \text { CATTGCTGACAGGATGCAGAAGG } & \text { TGCTGGAAGGTGGACAGTGAGG } \\ \text { Nfkbia } & \text { GGTGACTTTGGGTGCTGAT } & \text { CTTGGTAGGTTACCCTGTTGAC } \\ \text { Ddit3 } & \text { TCCTGTCCTCAGATGAAATTGG } & \text { GCAGGGTCAAGAGTAGTGAAG } \\ \text { Gadd45a } & \text { CTGTGTGCTGGTGACGAA } & \text { GCACCCACTGATCCATGTAG } \\ \text { Akt3 } & \text { CAGAACGACCAAAGCCAAATAC } & \text { CTTCCGTCCACTCTTCTCTTTC } \\ \text { Hyou1 } & \text { CGCAAAGTCATCACCTTTAACC } & \text { GTCAGATTCTGGGAGCCAAATA } \\ \text { Cflar } & \text { CTGATTATAGGGTCCTGCTGATG } & \text { TTGCCTCTGCCTGTGTAATC }\end{array}$

4.4 Statistical analysis

Scheffe and one-way ANOVA were used to analyze the statistical significance. A Pvalue less than 0.05 was considered to be statistically significant. Results were presented as means $\pm \mathrm{SD}\left({ }^{*} \mathrm{p}<0.05\right.$ and $\left.{ }^{* *} \mathrm{p}<0.001\right)$. The RNA-seq data were obtained as differences between treatment and control groups. DEGseq was used to analyze the statistical significance between treatment and control groups. Relative log expression was higher than 2 and the corrected p-value was less than 0.005 , which was considered to indicate statistical significance.

\section{Conclusions}

In conclusion, these findings show that Sarcodia suieae acetyl-xylogalactan could induce anti-apoptotic cflar gene expression and downregulate the expression of the apoptosis factors Ddit3, and Hyou1 to protect the heat stress-induced macrophage. According to these evidence, we presented that the extracted S. suieae acetyl-xylogalactan might directly induce Cflar gene expression but reduces Nfkbia, Hyou1, Ddit3, Gadd45a and $A k t 3$ expression, resulting in regulating apoptosis response via the intrinsic apoptosis signaling pathway (As Figure. $4 \mathrm{~B}$ ).

\section{Patents}

There is no patent resulting from the work reported in this manuscript. 


\begin{abstract}
Author Contributions:
Kuang-Teng Wang: Sample analysis and Data curation ;

Po-Kai Pan: Data curation, Formal analysis.

Tsung-Meng Wu and Yu-Sheng Wu: Data curation, Formal analysis and Article Writing.

Funding:
\end{abstract}

This research was supported from National Pingtung University of Science and Technology.

Institutional Review Board Statement:

Not applicable

Data Availability Statement:

The datasets generated during and analysed during the current study are available in the NCBI repository with the BioProject database as http://www.ncbi.nlm.nih.gov/bioproject/686826

\title{
Acknowledgments:
}

The authors would like to thank Dr. Yu-Lun Kuo at BIOTOOLS Co., Ltd in Taiwan for kindly supporting analysis of NGS data.

\section{Conflicts of Interest:}

The authors declare no conflict of interest.

\section{References}

1. [1] Ganesan, S.; Volodina, O.; Pearce, S.C.; Gabler, N.K.; Baumgard, L.H.; Rhoads, R.P.; Selsby, J.T. Acute heat stress activated inflammatory signaling in porcine oxidative skeletal muscle. Physiol. Rep. 2017, 5, e13397.

2. [2] Chen, L.; Deng, H.; Cui, H.; Fang, J.; Zuo, Z.; Deng, J.; Li, Y.; Wang, X.; Zhao, L. Inflammatory responses and inflammationassociated diseases in organs. Oncotarget 2018, 9, 7204-7218.

3. [3] Takeuchi, O.; Akira, S. Pattern recognition receptors and inflammation. Cells 2010, 140, 805-820.

4. [4] Zeng, T.; Li, J.J.; Wang, D.Q.; Li, G.Q.; Wang, G.L.; Lu, L.Z. Effects of heat stress on antioxidant defense system, inflammatory injury, and heat shock proteins of Muscovy and Pekin ducks: evidence for differential thermal sensitivities. Cell Stress Chaperones 2014, 19, 895-901.

5. [5] Montilla, S.I.R.; Johnson, T.P.; Pearce, S.C.; Gardan-Salmon, D.; Gabler, N.K.; Ross, J.W.; Rhoads, R.P.; Baumgard, L.H.; Lonergan, S.M.; Selsby, J.T. Heat stress causes oxidative stress but not inflammatory signaling in porcine skeletal muscle. Temp. 2014, 17, 42-50.

6. [6] Quinteiro-Filho, W.M.; Gomes, A.V.S.; Pinheiro, M.L.; Ribeiro, A.; Ferraz-De-Paula, V.; Astolfi-Ferreira, C.S.; Ferreira, A.J.P.; Palermo-Neto, J. Heat stress impairs performance and induces intestinal inflammation in broiler chickens infected with Salmonella Enteritidis. Avian Pathol. 2012, 41, 421-427.

7. [7] Furusawa, Y.; Yamanouchi, Y.; Iizumi, T.; Zhao, Q.L.; Mitsuhashi, Y.; Morita, A.; Enomoto, A.; Tabuchi, Y.; Kondo, T. Checkpoint kinase 2 is dispensable for regulation of the p53 response but is required for G2/M arrest and cell survival in cells with p53 defects under heat stress. Apoptosis 2017, 22,1225-1234.

8. [8] Ganesan, S.; Pearce, S.C.; Gabler, N.K.; Baumgard, L.H.; Rhoads, R.P.; Selsby, J.T. Short-term heat stress results in increased apoptotic signaling and autophagy in oxidative skeletal muscle in Sus scrofa. J Therm. Biol. 2018, 72, 73-80.

9. [9] Ganesan, S.; Summers, C.M.; Pearce, S.C.; Gabler, N.K.; Valentine, R.J.; Baumgard, L.H.; Rhoads, R.P.; Selsby, J.T. Short-term heat stress causes altered intracellular signaling in oxidative skeletal muscle. J Anim. Sci. 2017, 95, 2438-2451.

10. [10] Park, S.; Lim, Y.; Lee, D.; Elvira, R.; Lee, J.M.; Lee, M.R.; Han, J. Modulation of protein synthesis by eIF2 $\alpha$ Phosphorylation protects cell from heat stress-mediated apoptosis. Cells 2018, 7, 254.

11. [11] Sarkar, S.A.; Kutlu, B.; Velmurugan, K.; Kizaka-Kondoh, S.; Lee, C.E.; Wong, R.; Valentine, A.; Davidson, H.W.; Hutton, J.C.; Pugazhenthi, S. Cytokine-mediated induction of anti-apoptotic genes that are linked to nuclear factor kappa-B (NF- $\kappa \mathrm{B})$ signalling in human islets and in a mouse beta cell line. Diabetologia 2009, 52, 1092-1101.

12. [12] Wu, T.M.; Nan, F.H.; Chen, K.C.; Wu, Y.S. Sarcodia suieae acetyl-xylogalactan regulate RAW 264.7 macrophage NF-kappa B activation and IL-1 beta cytokine production in macrophage polarization. Sci. Rep. 2019, 9, 19627.

13. [13] Zhang, X.; Ren, A.; Li, M.J.; Cao, P.F.; Chen, T.X.; Zhang, G.; Shi, L.; Jiang, A.L.; Zhao, M.W. Heat stress modulates mycelium growth, heat shock protein expression, ganoderic acid biosynthesis, and hyphal branching of Ganoderma lucidum via cytosolic $\mathrm{Ca}^{2+}$. Appl. Environ. Microbiol. 2016, 82, 4112-4125.

14. [14] Slawinska, A.; Hsieh, J.; Schmidt, C.J.; Lamont, S.J. Heat stress and lipopolysaccharide stimulation of chicken macrophagelike cell line activates expression of distinct sets of genes. PLoS One 2016, 11, e0164575.

15. [15] Moine, L.; De-Barboza, G.D.; Pérez, A.; Benedetto, M.; De-Talamoni, N.T. Glutamine protects intestinal calcium absorption against oxidative stress and apoptosis. Comp. Biochem. Physiol. Part A Mol. Integr. Physiol. 2017, 212, 64-71. 
16. [16] Catozzi, C.; Ñvila, G.; Zamarian, V.; Pravettoni, D.; Sala, G.; Ceciliani, F.; Lacetera, N.; Lecchi, C. In-vitro effect of heat stress on bovine monocytes lifespan and polarization. Immunobiology 2020, 225, 151888.

17. [17] Yuan, M.; Meng, X.W.; Ma, J.; Liu, H.; Song, S.Y.; Chen, Q.C.; Liu, H.Y.; Zhang, J.; Song, N.; Ji, F.H.; Peng, K. Dexmedetomidine protects $\mathrm{H} 9 \mathrm{c} 2$ cardiomyocytes against oxygen-glucose deprivation/reoxygenation-induced intracellular calcium overload and apoptosis through regulating FKBP12. 6/RyR2 signaling. Drug Des. Dev. Ther. 2019, 2, 3137-3149.

18. [18] Li, L.; Su, Z.; Zou, Z.; Tan, H.; Cai, D.; Su, L.; Gu, Z. Ser46 phosphorylation of p53 is an essential event in prolyl-isomerase Pin1-mediated p53-independent apoptosis in response to heat stress. Cell Death Dis. 2019, 10, 96.

19. [19] Daigeler, A.; Brenzelm, C.; Bulut, D.; Geisler, A.; Hilgert, C.; Lehnhardt, M.; Steinau, H.U.; Flier, A.; Steinstraesser, L.; KleinHitpass Mittelkötte, U.; Uhl, W.; Chromik, A.M. TRAIL and Taurolidine induce apoptosis and decrease proliferation in human fibrosarcoma. J Exp. Clin. Cancer Res. 2008, 27, 82.

20. [20] Li, T.; Su, L.; Liu, X.; Zhang, Y.; Liu, X. DDIT3 and KAT2A proteins regulate TNFRSF10A and TNFRSF10B expression in endoplasmic reticulum stress-mediated apoptosis in human lung cancer cells. J Biol. Chem. 2015, 290,11108-11118.

21. [21] Lurlaro, R.; Muñoz-Pinedo, C. Cell death induced by endoplasmic reticulum stress. FEBS J 2016, 283, $2640-2652$.

22. [22] Syc-Mazurek, S.B.; Fernandes, K.A.; Wilson, M.P.; Shrager, P.; Libby, R.T. Together JUN and DDIT3 (CHOP) control retinal ganglion cell death after axonal injury. Mol. Neurodegener 2017, 12, 71.

23. [23] He, M.X.; He, Y.W. CFLAR/c-FLIPL: a star in the autophagy, apoptosis and necroptosis alliance. Autophagy 2013, 9, 791-793.

24. [24] Tsuchiya, Y.; Nakabayashi, O.; Nakano, H. FLIP the Switch: Regulation of Apoptosis and Necroptosis by cFLIP. Int. J Mol. Sci. 2015, 16, 30321-30341.

25. [25] Wang, Y.; Zhao, Y.; Zhang, A.; Ma, J.; Wang, Z.; Zhang, X. Targeting of miR-20a against CFLAR to potentiate TRAILinduced apoptotic sensitivity in HepG2 cells. Eur. Rev. Med. Pharmacol. Sci. 2017, 21, 2087-2097. 